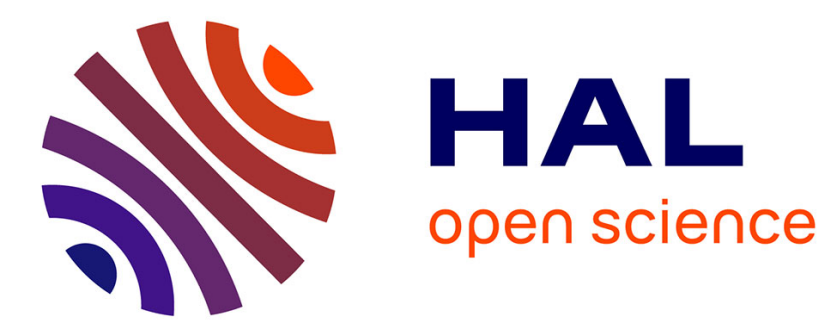

\title{
Time-resolved luminescence Z-scan of CsI using power femtosecond laser pulses
}

\author{
A. Belsky, N. Fedorov, S. Gridin, A. Gektin, P. Martin, D. Spassky, A. \\ Vasil'Ev
}

\section{- To cite this version:}

A. Belsky, N. Fedorov, S. Gridin, A. Gektin, P. Martin, et al.. Time-resolved luminescence Zscan of CsI using power femtosecond laser pulses. Radiation Measurements, 2019, 124, pp.1-8. 10.1016/j.radmeas.2019.02.021 . hal-02130412

\section{HAL Id: hal-02130412 \\ https://hal.science/hal-02130412}

Submitted on 22 Oct 2021

HAL is a multi-disciplinary open access archive for the deposit and dissemination of scientific research documents, whether they are published or not. The documents may come from teaching and research institutions in France or abroad, or from public or private research centers.
L'archive ouverte pluridisciplinaire HAL, est destinée au dépôt et à la diffusion de documents scientifiques de niveau recherche, publiés ou non, émanant des établissements d'enseignement et de recherche français ou étrangers, des laboratoires publics ou privés.

\section{(c) (1) $\$$}

Distributed under a Creative Commons Attribution - NonCommerciall 4.0 International 


\title{
Time-resolved luminescence Z-scan of CsI using power femtosecond laser
} pulses

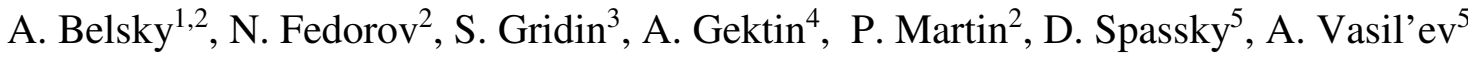 \\ ${ }^{1}$ Institute of Light and Matter, CNRS, University Lyon1, France \\ ${ }^{2}$ CELIA, Université de Bordeaux, CNRS, CEA, Talence, France \\ ${ }^{3}$ Department of Physics, Wake Forest University, Winston Salem, NC, USA \\ ${ }^{4}$ Institute for Scintillation Materials, Kharkov, Ukraine \\ ${ }^{5}$ Skobeltsyn Institute of Nuclear Physics, Moscow State University, Moscow, Russia
}

\begin{abstract}
Time-resolved Z-scan technique gives the opportunity to obtain consistent data for dependence of the yield and decay kinetics from excitation density. The excitation by $4^{\text {th }}$ harmonics of femtosecond Ti:Sapphire laser $(6.2 \mathrm{eV})$ allows to measure with wide dynamical range of excitation density (from $10^{17}$ to $10^{22}$ excitations per cubic centimeter) CsI luminescence. In the density range $10^{18}-10^{19} \mathrm{~cm}^{-3}$ the yield of $310 \mathrm{~nm}$ band (fast intrinsic luminescence) increases linearly with energy of the laser pulse, and yield of $430 \mathrm{~nm}$ band decreases inversely. In the same density range the subnanosecond decay of $310 \mathrm{~nm}$ band becomes slower and in microsecond kinetics of $430 \mathrm{~nm}$ band increases the contribution of nanosecond components.
\end{abstract}

\section{Introduction}

A high-energy ionizing photon or particle upon interaction with semiconductors or insulators creates a structured excited region with concentration of secondary electron-hole pairs ranging from $10^{10}$ to $10^{22}$ pairs per cubic centimeter. It is well-known that density of excitations controls the process of energy transfer, localization of excitations, scintillation yield and decay, storage of energy, etc. Excitation densities above $10^{17} \mathrm{~cm}^{-3}$ will be called high, since about this density one usually observes effects of interaction between electronic excitations. High excitation density could result not only in quenching of emission centers (excitons), but also can stimulate different processes of creation of new excited states with superlinear dependence of excitation density. Influence of excitation density on luminescence in semiconductor materials was carefully studied in Ref. [1]. Many interaction mechanisms between free excitons and various electronic excitations were established, along with their relaxation processes. Interaction of electronic excitations in ionic crystals with prompt selftrapping of excitons is investigated much less. Nonetheless, a lot of experimental data have 
been acquired in recent years, which relates to ionization of ionic crystals with radiation, especially with heavy particles. These processes involve interaction of electronic excitations, formation of defects and self-trapped excitons, which is typical for high local excitation density [2]. Systematic studies of the effects of high local excitation density in ionic crystals started right after development of powerful femtosecond lasers. The first study of luminescence kinetics in an ionic crystal at high excitation density was done on $\mathrm{CdWO}_{4}$ [3] under VUV excitation with a high harmonic of Ti:Sapphire laser. Analysis of the decay curves measured at several high excitation densities pointed to a dipole-dipole interaction mechanism of the self-trapped excitons. A Z-scan experiment using $3^{\text {rd }}$ and $4^{\text {th }}$ harmonics of Ti:Sapphire laser was used to investigate how emission intensity is affected by excitation density in a series of ionic crystals [4] in comparison to wide band gap semiconductors. Some typical patterns shown in this study are the following: (a) linear dependence of luminescence intensity on excitation density until a threshold about $10^{19}-10^{20}$ is reached, then quenching of luminescence is observed; (b) superlinear increase of luminescence intensity with density up to the threshold, emission quenching above the threshold (this result was interpreted in terms of the model of saturated surface losses). Depending on the process responsible for luminescence quenching, the intensity dependence on excitation density can be fitted in assumption that the quenching term in kinetic rate equations increases as square of concentrations (in case of exciton+exciton quenching) or as cube of concentration (in case of Auger process like electron+electron+hole quenching). In [4] these cases are regarded as $2^{\text {nd }}$ or $3^{\text {rd }}$ order quenching.

In this work we present experimental data on luminescence of wide-band-gap crystals excited by harmonics of a powerful femtosecond Ti:Sapphire laser. We study the relation between luminescence intensity and excitation density using time-resolved luminescence Zscan, the method which allows one to change excitation density in a wide dynamic range. This experiment allows creating excitation densities similar to those formed in different parts of ionization tracks in scintillators. High sensitivity to excitation density is known in several scintillation crystals with applied significance. One example is undoped CsI, which is the sample material in this work. To create electronic excitations in CsI crystal at $\mathrm{T}=300 \mathrm{~K}$, the threshold photon energy of $5.8 \mathrm{eV}$ is required to cross the energy gap. This value corresponds to the $4^{\text {th }}$ harmonic of the Ti:Sapphire laser $(6.2 \mathrm{eV})$ used in this study. We can simulate in CsI excitation conditions of different regions of ionizing particle's tracks by changing the density of such laser excitation using Z-scan technique.

There are usually two emissions generated in CsI under high energy excitation. One 
of them blue band (420-450 $\mathrm{nm}$ ) demonstrates about standard behavior typical for impurity related emission, with the first excitation band below the energy gap, and slightly depends of excitation density at low intensities. On the contrary, the intrinsic emission UV band peaking at $300-310 \mathrm{~nm}$ at room temperature exhibits strong unusual excitation spectrum and dependence on excitation density.

This band does not excited by low-intensity radiation (synchrotron radiation) by photons with energy 6 to $15 \mathrm{eV}$, i.e. above the threshold of fundamental absorption [5]. At the same time this ultraviolet band with fast nanosecond emission is effectively excited by ionizing radiation and some detectors of high-energy particles are based on this emission. We proposed [5] that the corresponding emission centers are created in the regions of high densities of excitations which are created by ionizing radiation.

The aim of the present paper is to continue the comparative study of the properties of luminescence bands of CsI crystal.

\section{Experimental}

The experiments were conducted in the CELIA laboratory (Bordeaux, France) and used a 1-TW Ti:Sapphire laser with pulse duration of $28 \mathrm{fs}$ and repetition rate of $1 \mathrm{kHz}$.

We measured the dependence of scintillation properties on excitation density using time resolved luminescence Z-scan as the method which allows one to change the excitation density in a wide dynamic range and therefore simulate the recombination processes in different parts of the ionizing particle track. The highest density of excitations is achieved under excitation by photons with energy higher than the energy gap, when the absorption depth is about a few tens of a nanometer. To achieve a required energy of $6.2 \mathrm{eV}(200 \mathrm{~nm})$, the $4^{\text {th }}$ harmonic was generated from the fundamental wavelength of the Ti:Sapphire laser using BBO crystals. Two $\mathrm{MgF}_{2}$ prisms were used to separate the $4^{\text {th }}$ harmonic. This spectral filter allowed us to reject the lower order harmonics.

The pulse energy of the laser was varied as necessary to assure stable harmonic generation. Samples were placed into a vacuum chamber. Gradual variation of the energy density falling onto the sample was done by translation of a focusing lens also placed in the vacuum chamber. The input window caused insignificant change of the beam profile. As the lens moved over $75 \mathrm{~mm}$ along $\mathrm{Z}$ axis, the laser spot size on the sample surface vas changing from $20 \mu \mathrm{m}$ to $450 \mu \mathrm{m}$ full width at half maximum (FWHM) (Fig. 1). Assuming Gaussian profile of the incident beam, excitation light intensity on the sample surface varied in the range from $1 \mu \mathrm{J} / \mathrm{cm}^{2}$ to $50 \mathrm{~mJ} / \mathrm{cm}^{2}$. This in turn caused changes in energy density per unit 
volume inside the sample. Given the short (about $20 \mathrm{~nm}$ ) penetration depth of the incoming light in the fundamental absorption range, the range of the laser spot sizes given above corresponds to maximum electronic excitation density in the sample from $10^{17}$ to $10^{22} \mathrm{~cm}^{-3}$.

Single crystals of CsI used in this experiment were grown in the Institute for Scintillation Materials (Kharkov, Ukraine) from purified raw material by the Stockbarger method. A $5 \times 5 \times 1 \mathrm{~mm}$ plate was cut and placed into the vacuum sample chamber. Luminescence light from the sample was focused into an optical fiber by a microscope objective. The other end of the fiber was connected to a TRIAX optical spectrometer. Two detectors were used after the spectrometer: an Andor CCD camera for spectrum measurements, and a Hamamatsu microchannel photomultiplier for pulse shape measurement.

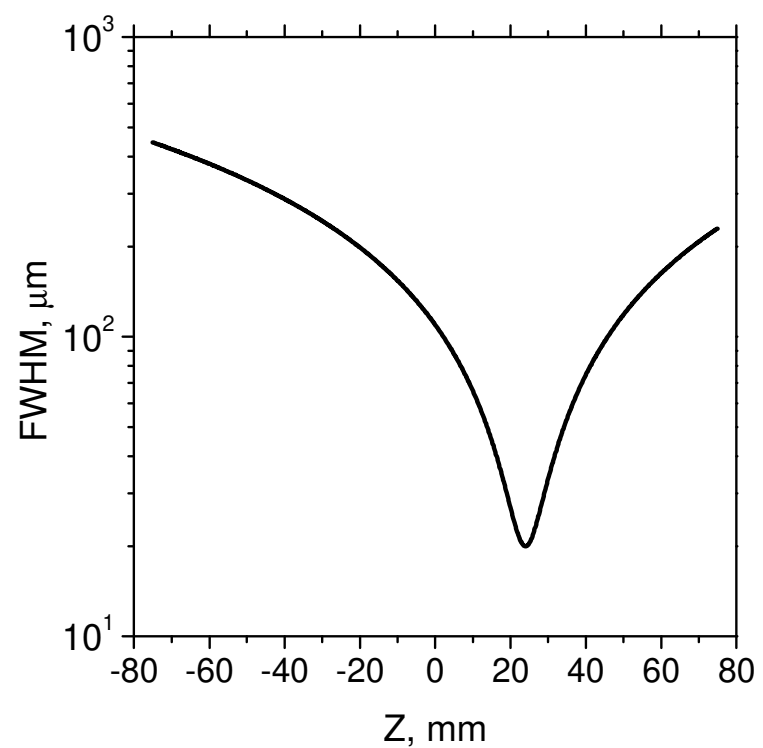

Fig.1. Variation of FWHM of laser spot on sample surface vs relative lens position $Z$.

\section{Experimental results and discussion}

\section{III.1 Emission spectra}

The emission spectrum of pure CsI under ionizing irradiation at $300 \mathrm{~K}$ usually consists of two bands (Fig. 2). In the studied sample blue band has maximum at about $430 \mathrm{~nm}$ (will be called here as $2.9 \mathrm{eV}$ band). It is known that in different CsI samples several bands peaked at $410,435,470$, or $530 \mathrm{~nm}$ are observed in 400 to $600 \mathrm{~nm}$ region [6]. All these bands correspond to luminescence of exciton complexes with intrinsic or impurity-based defects. Investigations performed with numerous series of activated, pure, deformed and annealed crystals lead to the conclusion [6,7] that the considering $2.9 \mathrm{eV}$ band is connected with 
oxygen-containing impurities. From VUV excitation spectra we conclude that formation of this excited state can be created both by consecutive capture of separated charge carriers, electron and hole, and by capture of an exciton. Under monochromatic UV excitation at low excitation density (by gas discharge lamps or synchrotron irradiation), this band has the first excitation maximum at around $5.0 \mathrm{eV}$, which is below the exciton absorption threshold in CsI. This observation confirms that formation of this excited state involves a defect with energy levels inside the energy gap. This observation also allows to propose the energy transfer from exciton to this defect center.

The UV band (300-310 $\mathrm{nm}$ ) usually identified as $4.1 \mathrm{eV}$ band is interpreted in [8] as room-temperature emission of self-trapped excitons (STE) which are localized due to the redistribution of the population of different exciton states with increase of temperature, together with the red shift of on-center exciton plus blue shift of off-center exciton which are observed in alkali halide crystals with $\mathrm{NaCl}$ structure (different from CsI one). This interpretation seems to be rather bold. It is known [9] that the emission of short-wavelength STE at $290 \mathrm{~nm}$ is totally quenched at $20 \mathrm{~K}$ under the excitation in exciton absorption range, whereas long-wavelength STE emission $(340 \mathrm{~nm})$ is quenched at 200K. Moreover, 4.1-eV band exhibits a number of properties different form STEs. The temperature dependence of the 4.1-eV band intensity has a maximum at about $200 \mathrm{~K}[8,10-12]$. The excitation spectrum of this band is also different from STE excitation spectra, those of cross luminescence or defectinduced luminescence. At $300 \mathrm{~K}$, the $4.1-\mathrm{eV}$ band is not excited at the exciton absorption range, the emission starts to grow only under an excitation of $20 \mathrm{eV}$ and above. This fact is explained in [8] by account for strong surface losses which are observed in fundamental absorption region due to the presence of near-surface defects. This explanation seems to be weak since the 4.1-eV excitation spectrum do not anticorrelate with absorption coefficient [5] (the absorption coefficient at 11 and $30 \mathrm{eV}$ is the same, but this emission is not observed at 11 $\mathrm{eV}$ excitation at all). Moreover, the growth of the yield of this band is superlinear with the excitation energy. At 50-eV excitation, this emission is almost 100 time brighter that what it is at 20-eV excitation [5]. Finally and most importantly for this study, in contrast to STE and impurity-related emission, the 4.1-eV band gets brighter at high excitation density [13]. We are calling this band 'fast intrinsic luminescence' (FIL) and provide discussion on its nature below in Section III.4. 


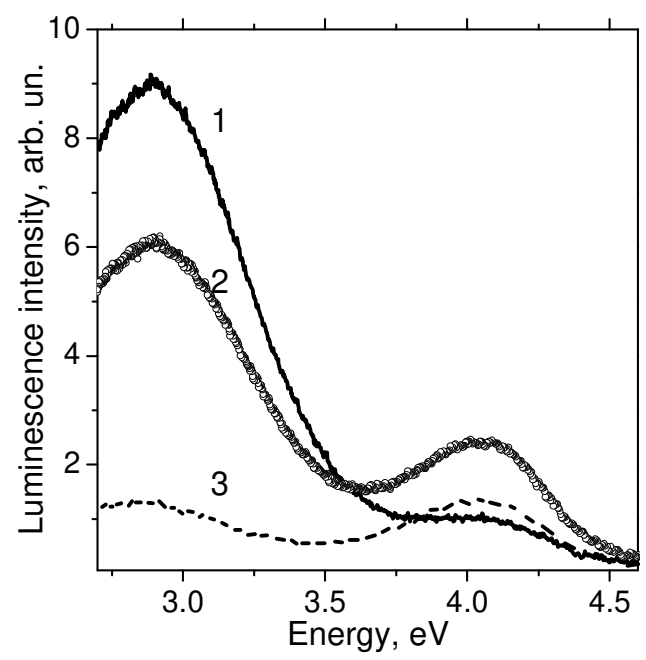

Fig. 2. Emission spectra of pure CsI under different excitation densities. Maximum densities in the excited domain are: $1-10^{17} \mathrm{~cm}^{-3}(\mathrm{Z}=-75 \mathrm{~mm}) ; 2-10^{18} \mathrm{~cm}^{-3}(\mathrm{Z}=-40 \mathrm{~mm}) ; 3-10^{20} \mathrm{~cm}^{-3}(\mathrm{Z}=15 \mathrm{~mm})$.

According to Fig. 2, the femtosecond laser pulses of $6.2 \mathrm{eV}$ used in this work excite both the 4.1-eV and 2.9-eV bands in pure CsI. The ratio between the two emission intensities depends on the excitation density. This density was changed by the shift of the focusing lens (Z-scan technique), the positions $\mathrm{Z}$ and the corresponding densities of electron excitations at the center of Gaussian spot near the sample surface are presented in the figure caption. For these estimations we use absorption coefficient for $6.2 \mathrm{eV}$ photons equals to $5 \times 10^{5} \mathrm{~cm}^{-1}$. At the lowest excitation density, $10^{17} \mathrm{~cm}^{-3}$, the $2.9-\mathrm{eV}$ band is dominant, while at the highest density, $10^{20} \mathrm{~cm}^{-3}$, the $4.1-\mathrm{eV}$ band is higher. The trends of excitation density dependence are opposite for these two bands. This dependence is discussed in details in Sec. III. The excitation density dependence observed here correlates with the opposite trends of excitation spectra of $2.9 \mathrm{eV}$ and $4.1 \mathrm{eV}$ bands observed at the X-ray absorption edges in CsI [14]. We suppose that relaxation of a core hole created by a photon with energy above the edge results in increased local density of the secondary electronic excitations. This effect is discussed in Sec. IV.

\section{III.2. Luminescence Z-scan: excitation density dependence}

Emission intensities for the two bands of CsI as a function of Z-position are shown in Fig. 3. Excitation pulse energy was varied in the range from $1 \mathrm{~nJ}$ to $30 \mathrm{~nJ}$. There are three curves for FIL and one curve for the 2.9-eV band shown in Fig. 3. 

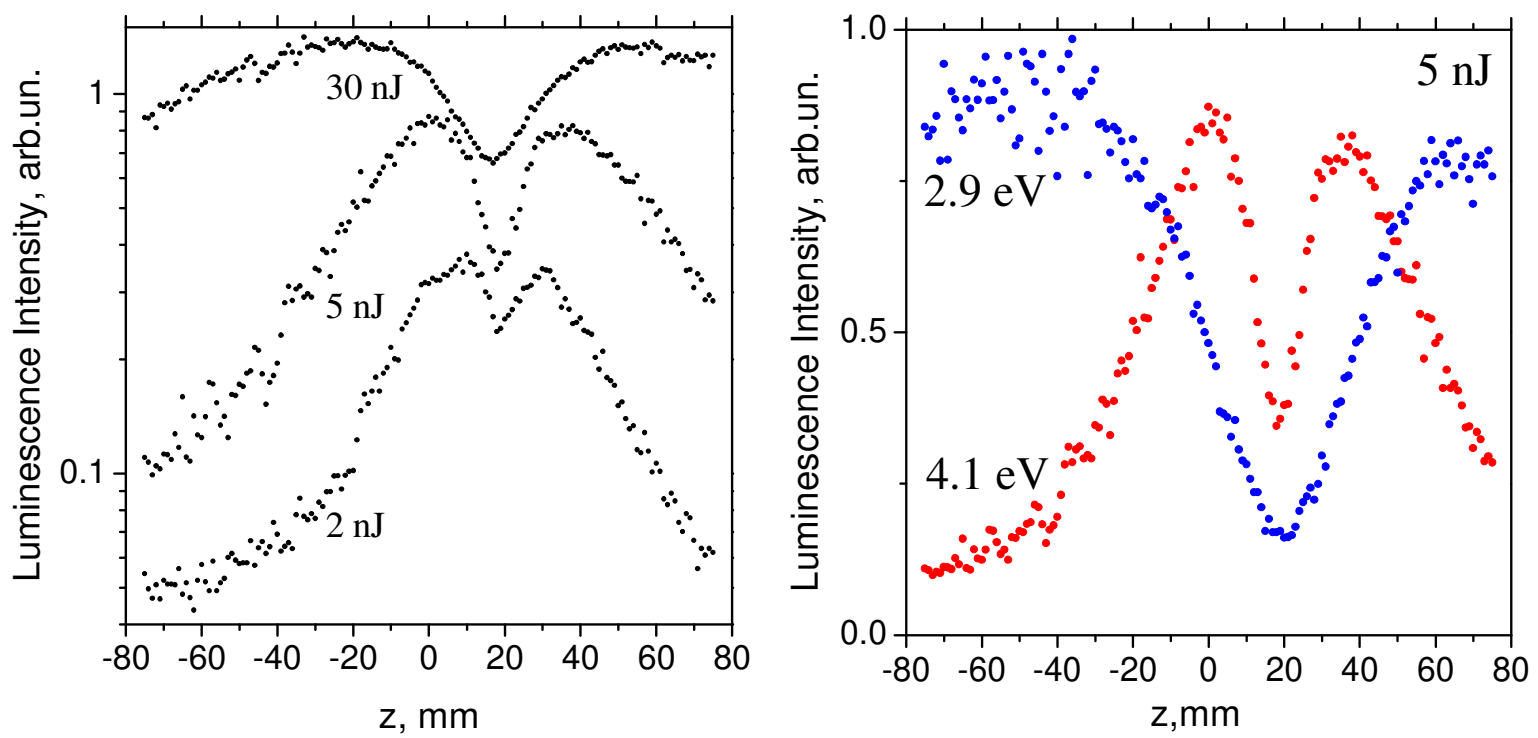

Fig.3 Left panel: Z-scan curves for FIL emission measured using laser beam of three pulse energies: 2 $\mathrm{nJ}, 5 \mathrm{~nJ}$ and $30 \mathrm{~nJ}$. Right panel: Z-scan curves for the two emission bands of CsI measured at $5 \mathrm{~nJ}$. The focal point of the lens is in the sample plane at $\mathrm{Z}=17 \mathrm{~mm}$.

At lower excitation densities, FIL intensity exhibits superlinear growth up to a certain density above which a decrease in intensity is observed. Such a dependence on excitation density for FIL in CsI was first reported in Ref. [4]. The corresponding Z-scan curve reported by Grim et al. is similar to the one obtained in this work at $5 \mathrm{~nJ}$. We also measured excitation density dependence for the 2.9-eV band as shown in Fig. 3, right panel. Comparing excitation density dependences for the two bands, they show opposite trends at lower excitation density. While the 2.9-eV emission first stays the same and then decreases when the focus approaches to the sample plane, the 4.1-eV FIL keeps growing until a certain density threshold is reached. Then quenching also gets into play. Such an unusual dependence versus excitation density for the FIL band points to a different origin of the centers responsible for the 4.1-eV emission. For reference, standard STE emission, activator luminescence, and cross luminescence all increase in proportion to the intensity of incident beam and do not show density dependence until a very high density is reached.

Based on the Z-scans measured in this work, we can calculate curves of luminescence intensity versus actual volume density of excitations. The relation between Z-position and the concentration of excitations at the spot center near the surface is given by the following equation:

$$
n_{\max }(Z)=\frac{4 \ln 2}{\pi} \frac{k J}{F W H M(Z)^{2} \hbar \omega},
$$


where $J$ is the energy of the pulse, $k$ is the absorption coefficient $\left(k=0.5 \times 10^{6} \mathrm{~cm}^{-1}\right)$, and $\hbar \omega$ $=6.2 \mathrm{eV}$. For instance, $1 \mathrm{~nJ}=6.242 \times 10^{9} \mathrm{eV} \rightarrow 1.007 \times 10^{9}$ photons of $6 \mathrm{eV}$. The full width at half of maximum for the spot is

$$
F W H M(Z)=\sqrt{2 \ln 2} w(Z), w(Z)=w_{0} \sqrt{1+\left(\frac{M^{2} \lambda Z}{\pi w_{0}^{2}}\right)^{2}}, w_{0}=\sqrt{f \lambda / \pi}
$$

Here $M^{2}$ is a beam quality factor modifying the Gaussian beam radius $w(Z), w_{0}$ is the beam waist radius, and $\lambda$ is the wavelength.

We have to note that the laser pulse produces strongly non-uniform distribution of concentration of excitations in the sample, namely approximately 2D Gaussian in surface and exponential in depth:

$$
n(x, y, z)=n_{\max }(Z) e^{-4 \ln 2\left(x^{2}+y^{2}\right) / F W H M^{2}(Z)-k z},
$$

where lowercase $z$ is the distance from the sample surface and $\mathrm{x}$ and $\mathrm{y}$ are distances from the spot center in the surface plane.

The theoretical simulation of the luminescence response is often made using rate equations for uniform distribution with $n(x, y, z)=$ const without account for non-uniform distribution of excitations in real experiment. Such conditions can be reproduced in experiment only for cases when absorption depth is larger than the sample thickness and the sample is illuminated uniformly. This is not the case for excitation in fundamental absorption region, since absorption depth is about few tens of nanometers. We can introduce the quantum yield of the luminescence $\eta_{l u m}(n)$ depending on uniform density $n$. The averaged yield of the luminescence in case of non-uniform excitation can be written as

$$
\left\langle\eta_{\text {lum }}\right\rangle=I_{\text {lum }} /(J / \hbar \omega)=\frac{\iiint n(x, y, z) \eta_{\text {lum }}(n(x, y, z)) d x d y d z}{\iiint n(x, y, z) d x d y d z} .
$$

This formula is correct if the drift length of the excitation is less than the typical dimensions of the excited region. The concentration of the excitations produced by the laser pulse changes relatively slowly in the XY sample plane. The steepest decrease is in $z$ direction into the crystal and the change is exponential with the typical penetration depth about $20 \mathrm{~nm}$. The distribution of excitations according to Eq. (3) depends on the maximum concentration $n_{\max }$ 
achieved near the surface at the center of the spot. Averaging the quantum yield over the 3D distribution (Eq. (3)) yield the following formula [3, 15]:

$$
\left\langle\eta_{\text {lum }}\right\rangle\left(n_{\max }\right)=\frac{1}{n_{\max }} \int_{0}^{n_{\max }} \eta_{\text {lum }}(n) \ln \frac{n_{\max }}{n} d n
$$

Therefore, in experiment we measure not the yield as the function of the initial concentration of excitations $\eta_{\text {lum }}(n)$ but the yield $\left\langle\eta_{\text {lum }}\right\rangle\left(n_{\max }\right)$ averaged over the distribution of initial concentrations which is characterized by maximal concentration $n_{\max }$. If we plot the series of Z-scan curves measured at different laser pulse energies $(2 \mathrm{~nJ}, 3.5 \mathrm{~nJ}, 6.5 \mathrm{~nJ}, 13.5 \mathrm{~nJ}$, $20 \mathrm{~nJ}$ ) in coordinates $\left\langle\eta_{\text {lum }}\right\rangle$ versus $n_{\max }$ (excitation density in the maximum of distribution achieved near the surface at the center of the spot, which is estimated using Eqs. (1) and (2) with $w_{0}=20 \mu \mathrm{m}$ ), the curves are not well coincided due to the beam energy measurement error. The curves for FIL and the 2.9-eV emission band after the correction of beam energies in frames of $10 \%$ are given in Fig. 4.

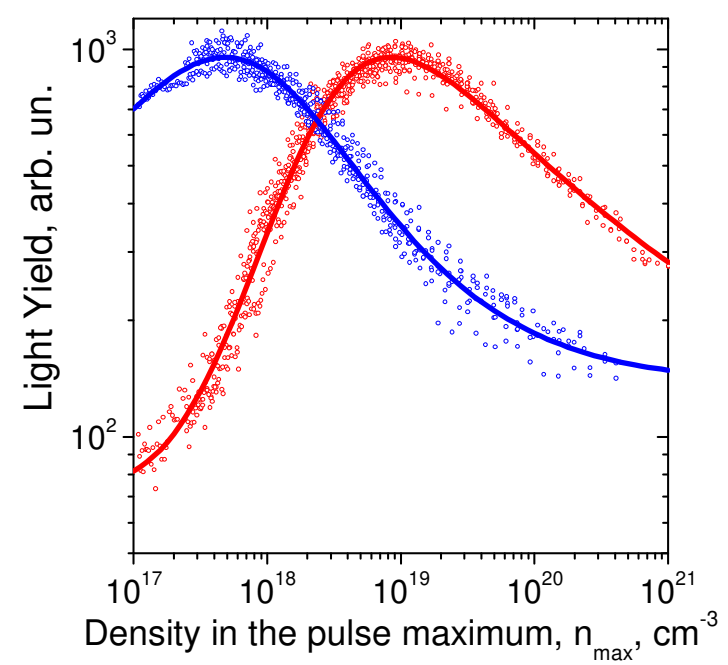

Fig.4 Averaged light yield $\left\langle\eta_{\text {lum }}\right\rangle\left(n_{\max }\right)$ the 2.9-eV (blue) and 4.1-eV FIL (red) bands as a function of the maximum excitation density induced by the laser. Solid lines correspond to the fitting procedure described in the text.

In order to obtain $\eta_{\text {lum }}(n)$ we have to solve the integral equation (5). Therefore, we fit the data presented in Fig. 4 in order to get analytical representations. The process is has some specifics, since concentrations and the yield changes in several orders of magnitude. We can fit $\ln \left\langle\eta_{\text {lum }}\right\rangle$ as a rational function of $x=\ln n_{\max }$ : 
$\ln \left\langle\eta_{\text {lum }}\right\rangle \approx \frac{a_{0}+a_{1} x+a_{2} x^{2}}{1+b_{1} x+b_{2} x^{2}+b_{3} x^{3}}$.

Corresponding fitting curves for the two bands are presented in Fig. 4 by solid lines.

Equation (5) can be solved and the local quantum yield $\eta_{\text {lum }}(n)$ can be obtained from the function $f\left(n_{\max }\right)=\left\langle\eta_{l u m}\right\rangle\left(n_{\max }\right)$ as

$\eta_{\text {lum }}(n)=\frac{d}{d n}\left(n \frac{d}{d n}(n f(n))\right)$

Therefore, we have to take second logarithmic derivative of the fitting curves presented by solid lines in Fig. 4. The result is shown in Fig. 5 for yield dependence of two bands.

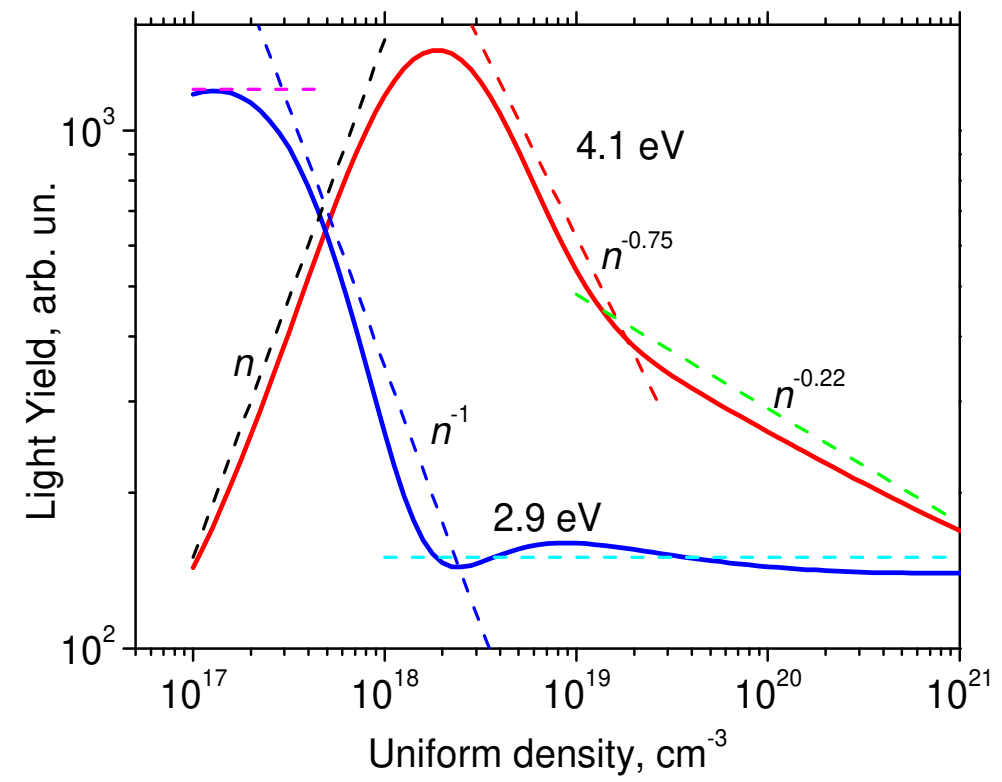

Fig. 5. Reconstructed dependence of luminescence LY $\eta_{\text {lum }}(n)$ of FIL (red) and $2.9 \mathrm{eV}$ (blue) bands as a function of uniform density $n$ of electronic excitations.

The non-monotonic behavior of the curves (see especially the valley in the 2.9-eVemission curve at about $2 \times 10^{18} \mathrm{~cm}^{-3}$ ) can be due to uncertainties in the numerical calculation of derivatives. These two emissions exhibit quite different dependences on excitation density. The quenching of $2.9 \mathrm{eV}$ emission starts at the concentrations when the mean distance between the excitations is about $20 \mathrm{~nm}$. In the same region of concentrations between $10^{17}$ $\mathrm{cm}^{-3}$ and $10^{18} \mathrm{~cm}^{-3}$, FIL emission increases about quadratically in intensity (which corresponds to a linear increase of the yield). The maximum of FIL yield is reached at the concentrations when the distance between excitations is about $8 \mathrm{~nm}$. This value is close to 
Onsager radius (the distance, at which the electrostatic potential of an electron is about thermal energy $k_{B} T$ ). The concentration of excitations at which the yield of FIL reaches its maximum is lower than the concentration values of $n_{\max }$ in the non-uniform case (compare with Fig. 4). This is because the average concentration $\langle n\rangle$ in case of 2D Gaussian and 1D exponential distribution of $n(x, y, z)$ is lower than the maximal concentration $n_{\max }$. The following increase of the concentration above $2 \times 10^{18} \mathrm{~cm}^{-3}$ results in the decrease of the FIL yield, mainly due to destroy of FIL centers after their interaction with others excitations. The products of this decay can be involved in reactions with defects, and therefore $2.9 \mathrm{eV}$ band do not decrease in this region.

\section{III.3. Variation of decay curves with increase of excitation density}

The 2.9-eV band undergoes strong quenching at pulse energy of $5 \mathrm{~nJ}$, as shown in Fig. 4. Figure 6 plots emission decay curves for this band under excitation by $15-\mathrm{nJ}$ pulses at $\mathrm{Z}$ positions: (1) at $\mathrm{Z}=17 \mathrm{~mm}$ (focus), when the beam size was $30 \mu \mathrm{m}$ (see fig.1), and (2) at $\mathrm{Z}=$ $-65 \mathrm{~mm}(420 \mu \mathrm{m})$. The decay profile is typical for radiative recombination at high density of electronic excitations [16].

In accordance with the excitation conditions curve 1 corresponds to the density of about $10^{18} \mathrm{~cm}^{-3}$, and curve 2 corresponds to $5 \times 10^{20} \mathrm{~cm}^{-3}$. The decay curves at initial $4 \mu \mathrm{s}$ can be approximated by exponents with characteristic times from 0.5 to $3 \mu \mathrm{s}$. After about $4 \mu \mathrm{s}$ both curves show the same behavior. Over $100 \mu$ s domain two curves demonstrate singleexponential decay with characteristic time of $33.5 \mu \mathrm{s}$, which can be regarded as radiation time of this center. In most publications characteristic times for this band are often between 1 and 3 $\mu \mathrm{s}[7,16]$. It should be noted that in these papers the decay kinetics for $430 \mathrm{~nm}$ band was measured within 3 to $5 \mu$ s interval under $\gamma$ or X-ray excitation. The characteristic time of this band under $\alpha$-particle excitation within the same interval is shorten down to hundreds of nanoseconds [17] which is connected with much denser excitation produced by $\alpha$-particles in comparison with X-ray quanta.

The kinetics of exciton and exciton-connected emissions under ionizing particle or energetic photon excitation usually consists of three domains. The initial part is characterized by accelerated kinetics due to interacting and quenching of excitations in regions with high concentration of them. The next interval has characteristic time close to radiation decay time and corresponds to emission from the regions of moderate concentration of excitations where recombination of electrons and holes into excitons is rather fast but the interaction of the 
products of recombination is weak. The last long-time domain is characterized by about hyperbolic tail spread up to seconds due to slow recombination of charge carriers in regions of low concentration of excitations together with their migration over traps. The majority of the published estimations corresponds to the first domain of the decay kinetics. The mentioned above radiation decay time of $33.5 \mu$ s corresponds to the second domain. If the whole interval of the decay recording is enlarged up to 1 to $10 \mathrm{~ms}$, the investigation of third domain becomes possible [18]. These measurements were out of the scope of our investigations.

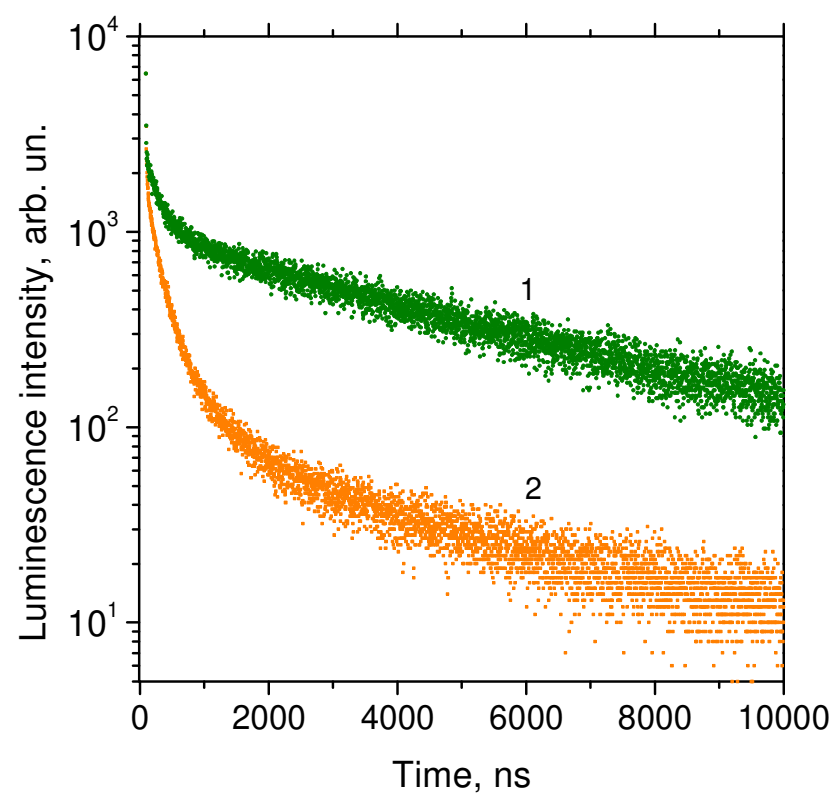

Fig. 6 Decay of the $2.9-\mathrm{eV}$ band at $\mathrm{n}_{\max }=10^{18} \mathrm{~cm}^{-3}(1-$ green dots $)$ and $5 \times 10^{20} \mathrm{~cm}^{-3}(2-$ orange dots).

The shapes of STE luminescence decay curves of $\mathrm{CdWO}_{4}$ under intense pulsed laser excitation with photon energy larger than the energy gap were analyzed in Ref. [3]. Since in ionic crystals excitons become self-trapped a few picoseconds after formation, a model of Auger-like dipole-dipole interaction of two STEs was applied as the primary quenching mechanism. STE concentration increases with excitation density, hence the distances between the particles become shorter. This facilitates interaction and quenching, the decay becomes faster. According to Fig. 6, the early part of the decay curve for the $2.9-\mathrm{eV}$ emission gets faster at higher excitation density. This emission band is attributed to an exciton trapped by a defect, possibly an oxygen-containing impurity [6]. The distance between such defects does not depend on the excitation density. This is why we assume that quenching of these excitations occurs because of interaction with the surrounding excitations which also grow in concentration. These excitations may be electrons, holes, or free excitons. Lifetime of these excitations is relatively short. Therefore quenching mostly takes place much faster than the 
lifetime of the 2.9-eV excitation. A more detailed analysis of the quenching process would require a simulation of the decay curves based on different quenching models. There could be several quenching mechanisms with different weights at different initial excitation density.

FIL decay is not monoexpoential but includes fast subnanosecond components. Fig. 7 shows how the decay profile changes versus excitation density. This change cannot be described by density quenching alone. The figure plots FIL decay curves from 0 to $40 \mathrm{~ns}$ at excitation densities from $10^{17}$ to $10^{20} \mathrm{~cm}^{-3}$. We know from previous section that FIL grows quadratically versus density $\mathrm{n}_{\max }$ in the range from $10^{17}$ to $10^{18} \mathrm{~cm}^{-3}$. This emission can also be observed at lower excitation densities. However, an excitation density threshold when the emission fully disappears was not found in this work. It is known form previous studies, that under monochromatic low-density excitation by a ${ }^{2} \mathrm{H}$ lamp or synchrotron irradiation FIL band is not excited at $300 \mathrm{~K}$.

Table 1 presents the parameters obtained from fitting the decay curves by two or three exponentials. This fitting does not allow one to make any conclusions concerning the mechanisms of emission, but gives the opportunity to compare these decays with ones obtained under ionizing radiation. The lowest excitation density used in this work was $10^{17}$ $\mathrm{cm}^{-3}$. In this case, a primary fast decay component of $350 \mathrm{ps}$ and some of slower 6-ns component constitute the curve (black curve in Fig. 7). The dots represent the experimental data and the solid lines are two-exponential (curve 1 and 2) and three-exponential (curves 3 and 4) fits.

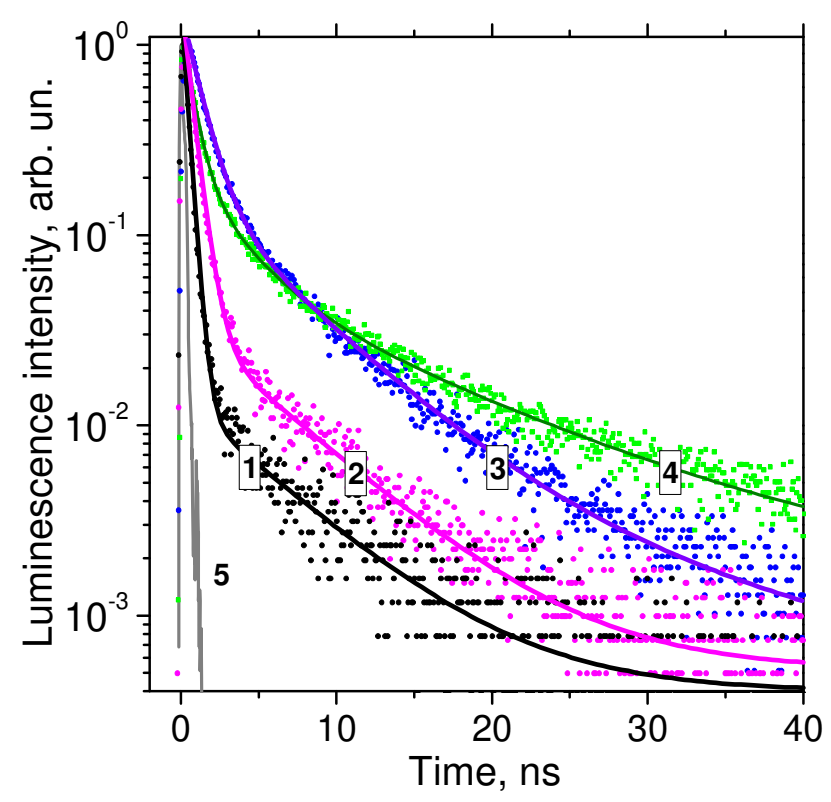


Fig. 7. FIL decay curves at different excitation densities $\mathrm{n}_{\max }: 1-10^{17} \mathrm{~cm}^{-3}, 2-10^{18} \mathrm{~cm}^{-3}, 3-10^{19} \mathrm{~cm}^{-}$ ${ }^{3}, 4-10^{20} \mathrm{~cm}^{-3}, 5$ - аппаратная функция детектора. Dots correspond to experimental data, solid lines are fits by 2 or 3 exponentials.

Inspection of curves 1, 2, and 3 in Fig. 7 shows that increase of the excitation density $n_{\max }$ up to $10^{19} \mathrm{~cm}^{-3}$ leads to faster decay, as the fast component slows down to $1 \mathrm{~ns}$ and the relative fraction of the slower 5-ns component increases. Note the appearance of a new 10-20-ns slow component at higher excitation density. Such excitation density dependence of FIL is hard to explain not having a complete model of the emission center. One can see however, that not only does the probability of FIL center formation increases with excitation density, but also the lifetime of these centers gets longer. This effect is possible if more stable emission centers are created with increase of excitation density. It is also possible that the lifetime of a single center does not increase, but still the increase of their concentration leads to a slower luminescence decay. This can happen if formation of FIL centers is distributed in time according to the lifetime of the high excitation density regions. The lifetime increase in this case is expected to saturate. One reason is the high-density quenching, i.e. quenching of the emission centers interacting with the other electronic excitations around them. Such interaction results in quenching at the early stage of the decay. Curve 4 in Fig. 7 decays faster than curve 3, which means that increase of excitation density $n_{\max }$ from $10^{19}$ to $10^{20} \mathrm{~cm}^{-3}$ starts density quenching of the FIL centers. The same acceleration of the decay was observed under analogous excitation densities in [19]. It is noted that the tail of curve 4 decays slower than the slow components of all the other curves. Therefore, at this high density formation of stable FIL centers is still ongoing.

Table 1 Approximation of FIL decay curves from figure 7 by 2 or 3 exponential function.

\begin{tabular}{|l|l|l|l|l|l|l|}
\hline $\begin{array}{l}\text { Density, } \\
\mathrm{cm}^{-3}\end{array}$ & $\mathrm{t} 1, \mathrm{~ns}$ & $\%$ & $\mathrm{t} 2$ & $\%$ & $\mathrm{t} 3$ & $\%$ \\
\hline $10^{17}$ & 0.36 & 88.4 & 6.0 & 11.6 & - & - \\
\hline $10^{18}$ & 0.60 & 76.6 & 6.07 & 23.4 & - & - \\
\hline $10^{19}$ & 1.08 & 49.7 & 5.33 & 42.6 & 15.1 & 7.7 \\
\hline $10^{20}$ & 0.68 & 31 & 2.87 & 27 & 11.7 & 42 \\
\hline
\end{tabular}

We have to note that published data have significant spread. One of the earlier investigations of decay kinetics of pure CsI under $\alpha$-particle and X-ray excitations mentioned two intervals of FIL characteristic times, 1-5 ns and 10-30 ns. Another publication [20] where electrons were used for excitation, gives two characteristic times - 10 and 36 ns. The comparison of 
these data with Tab. 1 shows that decay kinetics can depend on the typical densities of excitations in each case, which are different for different ionizing particles and their energies.

Now let us look at the 2.9-eV and FIL decay curves in a wider time frame (see Fig. 8). We can a better sense of the slow decay component of FIL as a function of density. FIL decay kinetics is overall much faster than the decay of the $2.9-\mathrm{eV}$ band. Only at the intensities about $10^{-3}-10^{-4}$ there is a slow component similar to the $2.9-\mathrm{eV}$ decay.

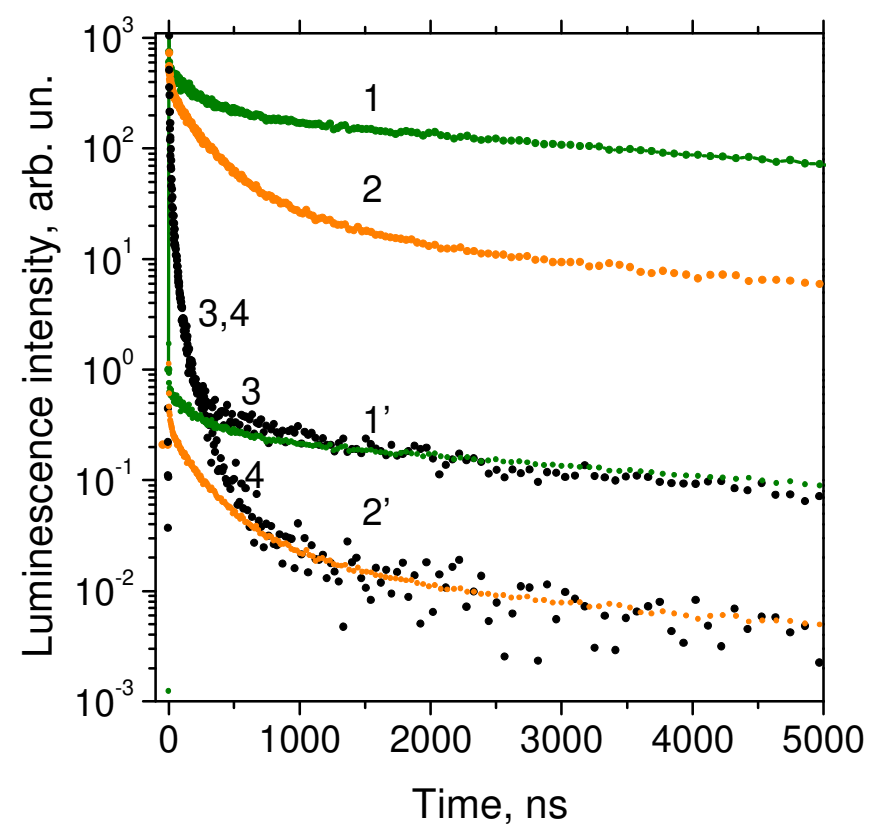

Fig. 8.1 and 2 - decay of $2.9 \mathrm{eV}$ band, 3 and $4-$ decay of the FIL band, excited by $15-n \mathrm{~J}$ laser pulses at $10^{18} \mathrm{~cm}^{-3}$ for curves 2 and 4 , at $5 \times 10^{20} \mathrm{~cm}^{-3}$ for curves 1 and 3 . For comparison, curve 1 and 2 were superimposed with curves 3 and 4 , the result is the curves 1 ' and 2'.

Figure 8 also shows decay curves for the $2.9-\mathrm{eV}$ band (the same curves as plotted in Fig. 6) at excitation densities $n_{\max }$ of $10^{18}$ and $5 \times 10^{20} \mathrm{~cm}^{-3}$. These curves were processed to reduce the noise at longer decay times. The technique we used to measure decay curves employs constant time step. The time step we chose was small enough to resolve the early fast part of the curve. Consequently, the number of counts in channel after about $200 \mathrm{~ns}$ was pretty small although the number of channels was high. We averaged the data in several channels over a time variable time interval which increased at higher time values. The time window was increased logarithmically with increase of time. This allowed for a significant improvement of signal quality and noise reduction at longer times. In Fig. 8 decay curves for 2.9-eV band and FIL at $\mathrm{t}>500 \mathrm{~ns}$ are selected to have the same excitation density. One can 
see that the FIL decay tails correspond to the slow decay of the 2.9-eV band. As shown in Fig. 2 , the two bands have some overlap. At a low excitation density, the band at $2.9 \mathrm{eV}$ is brighter than FIL. Therefore, the microsecond decay of the 2.9-eV emission contributes to the 4.1-eV

FIL decay. It is noted that the fraction of the tail component in FIL is higher at a lower density of excitation. To eliminate the actual slow component of FIL irrelevant to the 2.9-eV emission, the tow curves are superimposed for direct comparison in Fig. 8. This procedure reveals some 50-100 ns component left in FIL. This is the decay component which increases with increase of excitation density as described above. It is an important finding that slower decay components, $100 \mathrm{~ns}$ and above, are not present in FIL radiative decay.

\section{III.4. The role of the mobility of electronic excitations}

The mobility of non-relaxed electrons, holes and excitons in CsI is rather high. The mobility of electrons was estimated as $\mu_{e}=8 \mathrm{~cm}^{2} / \mathrm{V} \mathrm{s}$ at room temperature [21]. According to the Einstein formula, $\mu=e D / k_{B} T$, the diffusion coefficient can be estimated. In this case $D_{e}=0.21 \mathrm{~cm}^{2} / \mathrm{s}$. The diffusion coefficient for holes is about an order of magnitude less than that for electrons, therefore diffusion coefficient for both holes and free excitons can be estimated as $D_{h} \sim D_{e x} \sim(0.02-0.1) \mathrm{cm}^{2} / \mathrm{s}$.

The diffusion coefficient for free carriers plays two roles. First of all, high values of the diffusion coefficient result in fast delivery of free carriers as reagents of different reactions, for instance quenching reaction for excited defect states, and possible reactions between different types of excitons (FE+FE, FE+STE). The reactions between excitations depend on concentration of reagents and their diffusion coefficients. Most of reactions are bimolecular one, when two reagents are converted into new one. The examples are creation of an exciton from an electron and a hole, energy transfer from exciton to defect (e.g. activator), interaction of two excitons into hypothetical new stable state ("exciton fusion" in terms of Ref. [22]). These reactions $A+B \rightarrow C$ between reagents $A$ and $B$ with production of reagent $C$ is represented in rate equations as terms $\beta_{A B} n_{A}(t) n_{B}(t)$ : 


$$
\begin{aligned}
& \frac{d n_{A}(t)}{d t}=\ldots-\beta_{A B} n_{B}(t) n_{A}(t)-\ldots, \\
& \frac{d n_{B}(t)}{d t}=\ldots-\beta_{A B} n_{A}(t) n_{B}(t)-\ldots, \\
& \frac{d n_{C}(t)}{d t}=\ldots+\beta_{A B} n_{A}(t) n_{B}(t)-\ldots
\end{aligned}
$$

where for diffusion-controlled reactions $\beta_{A B}=4 \pi\left(D_{A}+D_{B}\right) R_{A B}, n_{i}$ is the concentration of the reagent $i, D_{i}$ is its diffusion coefficient, and $R_{A B}$ is the "capture" radius for the reaction. For reaction of oppositely charged carriers $R_{A B}$ equals to Onsager radius $e^{2} / 4 \pi \varepsilon_{0} \varepsilon k_{B} T$ (if we neglect screening) and is about $10 \mathrm{~nm}$ for room temperature. In case of dipole-dipole energy transfer with characteristic radius $R_{d-d, A \rightarrow B}$ this "capture" radius equals to $R_{c}=0.91 R_{d-d, A \rightarrow B}\left(\frac{R_{d-d, A \rightarrow B}^{2}}{\left(D_{A}+D_{B}\right) \tau_{A}}\right)^{1 / 4}$

The second role of the high diffusion coefficient is important when the crystal is excited in the fundamental absorption range. In this case the attenuation length for excitation light is about few tens of nanometers $\left(20 \mathrm{~nm}\right.$ in case of the excitation of CsI by $4^{\text {th }}$ harmonics of Ti:Sapphire laser). Due to diffusion the distribution of carriers is spread into the crystal as $\sqrt{D t}$. For high concentrations the diffusion of electrons and holes is ambipolar and we should take $D_{h}$ as diffusion coefficient. For $D_{h}=0.02 \mathrm{~cm}^{2} / \mathrm{s}$ the depth of the distribution of excitations increases up to $45 \mathrm{~nm}$ during first nanosecond after excitation and up to $1.4 \mu \mathrm{m}$ during first microsecond. Therefore, the concentration of excitations rapidly decreases even without any other channels of relaxation of free excitation. This process depends on the type of the reactions between free carriers at the surface. In case of reflection of excitations from the surface (no surface losses) the total number of free excitations integrated over the direction inside the crystal does not change due to diffusion. In case of the high rate of recombination of electrons and holes (or non-radiative death of excitons) the total number of excitations decreases rapidly during first nanoseconds. The effect of the penetration of excitation into the crystal interior is probably visualized in the decay kinetics of 2.9-eV emission. The dependence of the decay on the excitation intensity is rather specific (see Fig. 6). The quenching is totally finished at $1 \mu$ s for lower intensity of excitation and at $2 \mu \mathrm{s}$ for higher intensity. This abrupt termination of the quenching can be explained by the escape of free carriers from the region where excited defect states have been initially created. 
Let us consider reactions between two excitons which can result in creation of new stable state ("fusion" reaction). If we take "fusion" radius equal to $2 \mathrm{~nm}$, the rate of the reaction with which excitons are involved in the reaction (inverse coefficient at $n_{e x}$ in rate equations) equals to $\beta_{e x+e x} n_{e x}=8 \pi D_{e x} R_{e x+e x} n_{e x}=5 \times 10^{-7} \mathrm{~cm}^{3} / \mathrm{s} \times n_{e x}$. For $n_{e x}=10^{18} \mathrm{~cm}^{-3}$ the typical reaction time is about 2 ps. The self-trapping time for holes and excitons in CsI is about few picoseconds at low temperatures [24]. Therefore, the interaction between non-relaxed excitons can form a new stable state in such "fusion" reaction, since the excess energy can be partially transformed into strongly perturbed state of ions surrounding the remaining exciton. The concentration of the resulting "fused" centers increases as the square of exciton concentration: $n_{f}(t, z)=\int_{0}^{t} \beta_{e x+e x} n_{e x}^{2}\left(t^{\prime}, z\right) d t^{\prime}$ (in case when the decay time of these centers is longer than $\left(\beta_{e x+e x} n_{e x}\right)^{-1}$; this is adequate supposition for concentrations of excitons higher than $n_{e x}=2 \times 10^{15} \mathrm{~cm}^{-3}$, which correspond to reaction time about $\left.1 \mathrm{~ns}\right)$. The increase of the concentration of FIL centers depends not only the reaction constant but on the decrease of the exciton concentration due to diffusion both to the crystal surface and to the interior of the sample. The mentioned above decrease of exciton concentration due to diffusion shows that the production of centers stops at few nanoseconds. The inverse decay time for these "fused" centers is a sum of the inverse radiation decay time, inverse intracenter non-radiation time and the rate of quenching of the "fused" centers in Auger-type reactions with excitons, electrons and holes. The quadratic behavior of this reaction corresponds to the rising part of the excitation density dependence of FIL (for concentrations below $2 \times 10^{18} \mathrm{~cm}^{-3}$, see Fig. 5), whereas Auger quenching is responsible for the falling part of this dependence (above $2 \times 10^{18}$ $\left.\mathrm{cm}^{-3}\right)$.

The reaction between exciton and defect with creation of excited state of the defect has rate of exciton decrease which depends linearly on defect concentration and does not depend on exciton concentration: $\beta_{e x \rightarrow d} n_{d}^{0}=4 \pi D_{e x} R_{e x \rightarrow d} n_{d}^{0}$. The increase of the number of excited defect centers $n_{d}(t, z)=\int_{0}^{t} \beta_{e x \rightarrow d} n_{e x}\left(t^{\prime}, z\right) n_{d}^{0} d t^{\prime}$ linearly depends on exciton concentration (again in case when the decay time of these centers is longer than $\left(\beta_{e x \rightarrow d} n_{d}^{0}\right)^{-1}$ ). The depletion of exciton concentration due to the diffusion to the surface also decreases the accumulation of defect centers. For small excitation intensities the yield of excited defect centers is constant (as shown in Fig. 5; the number of such centers is proportional to laser 
intensity). At higher intensities Auger processes quench the emission of such centers. This quenching occurs at concentrations significantly less than for most other crystals, since the diffusion coefficient for free carriers is much higher for CsI than for other crystals. The quenching of defect centers occurs at lower intensities than the quenching of FIL, since the lifetime of such centers is much longer than that for "fused" centers responsible for FIL.

\section{Application to core level excited luminescence}

It is known that photons with energies below and above the core absorption edge produce different energy distribution and therefore different spatial distribution of electronic excitations. Namely, the Auger relaxation of core hole which is produced by photons with energy above the threshold results in creation of region with high concentration of holes. Excitation spectra for $2.9 \mathrm{eV}$ and FIL bands in the spectral region near $\mathrm{L}_{3}$ edge of iodine (Fig. 9) were measured at the first time at EXAFS station in LURE [14]. Taking into account the significance of this result for the topic of the present paper and shortage of the discussion in [14], we discuss this result in more details.

The excitation spectrum for 2.9-eV band near the absorption core edge have peculiarities which are opposite to that of the spectrum of $\mathrm{X}$-ray $\mathrm{L}_{3}$ fluorescence, which in turn is proportional to partial absorption coefficient for this core level. But this is not the mirror reflection of peculiarities, the amplitude of the structure in excitation spectrum is much higher than the amplitude of the fluorescence excitation spectrum. This fact probably reflects the nonlinear dependence of the luminescence yield on the absorption coefficient. Note that this is the one of the reasons which makes XEOL (X-ray Excited Optical Luminescence) EXAFS not very informative. The luminescence just above absorption edge decreases at about $40 \%$ in comparison with the level below $\mathrm{L}_{3}$ edge. Figure 5 shows that $2.9 \mathrm{eV}$ emission yield decreases when the concentration of excitations $5 \times 10^{17} \mathrm{~cm}^{-3}$, which can explain strong sensitivity of this emission to the energy of the photon, since photons with energy above the core level threshold create regions with high densities of excitations due to Auger relaxation of $\mathrm{L}_{3}$ core hole. We can estimate that the mean concentration of excitations in these regions reaches $10^{19} \mathrm{~cm}^{-3}$.

Usually X-ray luminescence excitation spectra exhibit a dip (rapid decrease) of the signal at the core absorption edges, which applies for both excitonic and activator luminescence [25]. Given that the absorption coefficient in these regions does not exceed $10^{4}$ 
$\mathrm{cm}^{-1}$, those dips are hard to explain by surface quenching. That would require an assumption about efficient diffusion of the electronic excitations toward the surface, where the particles would have to travel hundreds and even thousands of nanometers. This assumption would require complete quenching of the luminescence excited by VUV photons at the fundamental absorption range, where the absorption coefficient is even higher. This is in contradiction with experimental observations. Therefore, the excitation intensity drop at the energy of the core level can be explained by quenching of electronic excitations (excitons and activator emission centers) at high local excitation density [16]. Such quenching can go by Auger mechanism when the distance between the electronic excitations is smaller than the radius of dipoledipole interaction. We assume that the 2.9-eV luminescence quenching with excitation density increase deals with a similar interaction of the excited emission center with another electronic excitation in its proximity.

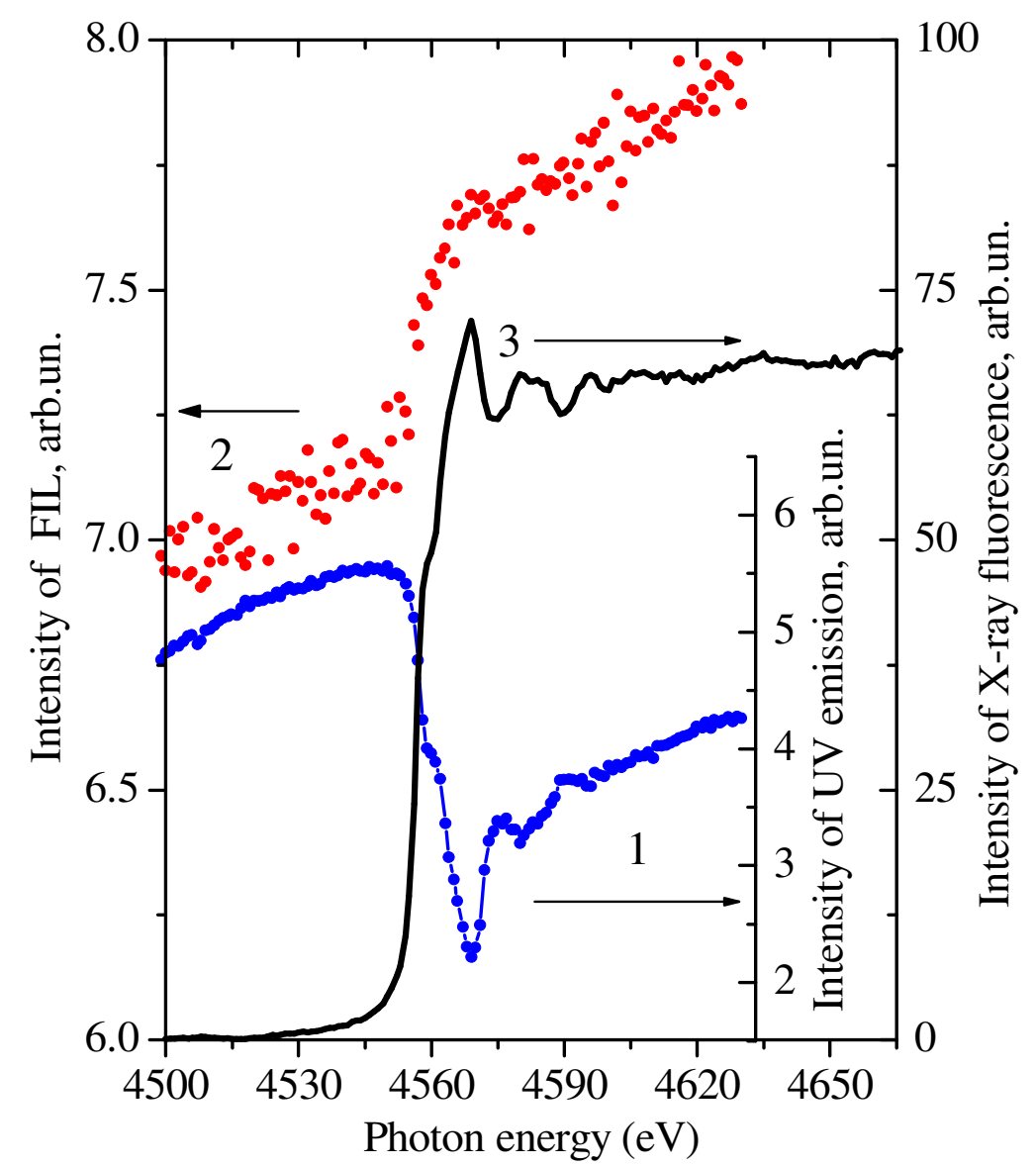

Fig 9. Luminescence excitation spectra of FIL (dots 2, left axis) and $2.9 \mathrm{eV}$ band (curve 1, small right axis) in CSI in the region of $\mathrm{L}_{3}$ edge of I and excitation spectrum of corresponding X-ray fluorescence (curve 3). 
The observed positive intensity step of the FIL band under excitation at the $\mathrm{L}_{3}$ edge of iodine (fig.9) is about $10 \%$, which is close to the ratio of partial absorption from this core level to the total absorption. Excitation of this core level results in a relaxation cascade of several electron-hole pairs with the initial radius $r<1 \mathrm{~nm}$. The high local excitation density created in this process, about $10^{20} \mathrm{~cm}^{-3}$, can be the reason of the FIL intensity increase. Based on the features of $2.9 \mathrm{eV}$ excitation spectrum, we propose above that the concentration of excitations can exceed $10^{19} \mathrm{~cm}^{-3}$. For these concentrations two processes compete: increased creation of FIL centers and their decay due to interaction of close centers. This competition can be the reason that positive step of FIL emission is not so prominent at $\mathrm{L}_{3}$ iodine edge. The detailed analysis of these data demands the development of more elaborated model for FIL centers and methods of calculation of spatial distribution of excitations accounting for Auger processes.

\section{Conclusion}

Time-resolved Z-scan technique gives the opportunity to obtain consistent data for dependence of the yield and decay kinetics in CsI for two emission bands: $4.1 \mathrm{eV}$ (Fast Intrinsic Luminescence) and $2.9 \mathrm{eV}$. The excitation by $4^{\text {th }}$ harmonics of femtosecond Ti:Sapphire laser $(6.2 \mathrm{eV})$ allows to measure with wide dynamical range of excitation density from $10^{17}$ to $10^{22}$ excitations per cubic centimeter. The luminescence intensity of $4.1 \mathrm{eV}$ band increases quadratically with increase of excitation density from $10^{17}$ to $10^{18} \mathrm{~cm}^{-3}$, whereas intensity of $2.9 \mathrm{eV}$ band does not increase at all in the same range (the yield of FIL emission increases linearly with energy of the laser pulse, and yield of $2.9 \mathrm{eV}$ band decreases inversely). In the density range $10^{18}-10^{19} \mathrm{~cm}^{-3}$ the sub-nanosecond decay of FIL band becomes slower and in microsecond decay of $2.9 \mathrm{eV}$ band increases the contribution of nanosecond components.

The quenching of $2.9 \mathrm{eV}$ band is naturally connected with interaction of the defectbound exciton with free electrons or excitons, concentration of which increases with increase of excitation density, and falls down in few tens of nanoseconds due to diffusion of excitations into the interior of the crystal. For $4.1 \mathrm{eV}$ band the model of the emission center can be connected with a new type of localized state of singlet exciton created through the "fusion" of self-trapped exciton and free exciton. Such scheme was proposed by K. Tanimura 
and N. Itoh [22] for KI and demands the collision of two excitons, the quadratic process on concentration of excitations.

\section{Aknowledgements}

The measurements at CELIA were performed with financial support of LaserlabEurope - The Integrated Initiative of European Laser Research Infrastructures (https://www.laserlab-europe.eu), project CNRS-CELIA002338. This research is supported by a European Union's Horizon 2020 research and innovation program under the Marie Skłodowska-Curie grant agreement No 644260 (INTELUM) and COST Action TD1401 (FAST). The financial support of these projects is greatly acknowledged.

\section{References}

[1] C. Klingshirn, Semiconductor Optics, Springer-Verlag Berlin Heidelberg, (2012).

[2] N. Itoh and M. Stoneham, Materials Modification by Electronic Excitation, 536 p., Cambridge University Press, (2000).

[3] M. Kirm, V. Nagirnyi, E. Feldbach, M. DeGrazia, B. Carre, H. Merdji, S. Guizard, G. Geoffroy, J. Gaudin, N. Fedorov, P. Martin, A. Vasil'ev, and A. Belsky, Phys. Rev. B 79 (2009) 233103.

[4] Q. Grim, K. B. Ucer, A. Burger, P. Bhattacharya, E. Tupitsyn, E. Rowe, V. M. Buliga, L. Trefilova, A. Gektin, G. A. Bizarri, W. W. Moses, and R. T. Williams, Phys. Rev. B 87 (2013) 125117.

[5] A. N. Belsky, A. N. Vasil'ev, V. V. Mikhailin, A. V. Gektin, P. Martin, C. Pedrini, D. Bouttet, Phys. Rev. B49 (1994) 13197

[6] N. V. Shiran, T. A. Charkina, V. I. Goreletsky, S. I. Vasetsky, A. I. Mitichkin and V. V. Shlyahturov. Tracks Radiat. Meas., 21 (1993) 107.

[7] S. Keszthelyi-Làndori, I. Földvàri , R. Voszka, Z. Fodor, Z. Seres. NIM A303 (1991) 374

[8] H. Nishimura, M. Sakata, T. Tsujimoto, and M. Nakayama, Phys. Rev. B 51 (1995) 2167.

[9] H. Lamatsch, J. Rossel, and E. Saurer. Phys. Status Solidi B 41 (1970) 605.

[10] M. Abdrakhmanov, S. Chernov, R. Deich and V. Gavrilov, J. of Luminescence 54 (1992) 197. 
[11] A. N. Belsky, A. N. Vasil'ev, V. V. Mikhailin, A. V. Gektin, N. V. Shiran, A. L. Rogalev, E. I. Zinin, Rev. Sci. Instrum. 63 (1992) 806

[12] A.V. Gektin, N.V. Shiran, A.N. Belskiy and A.N. Vasil'ev, NuclTracksRadMeasur 21, 11(1993)

[13] S.Sebban, P.Jaeglé, G.Jamelot, A.Carillon, A.Klisnick, P.Zeitoun, F.Albert, D.Ros, A.N.Belsky, I.A.Kamenskikh, B.Rus, Inst.Phys.Conf.Ser. 151: 528, IOP Publishing Ltd (1996)

[14] A. N. Belsky, R. Cortes, A. V. Gektin, P. Martin, V. V. Mikhailin, C. Pedrini, J. of Luminescence 72-74 (1997) 93

[15] A. N. Vasil'ev, IEEE TNS, 55 (2008) 1054.

[16] A. N. Belsky, R. A. Glukhov, I. A. Kamenskikh, P. Martin, V. V. Mikhailin, I. H. Munro, C. Pedrini, D. A. Shaw, I. N. Shpinkov, A. N. Vasil'ev, Journal of Electron Spectroscopy and Related Phenomena 79 (1996) 147.

[17] P. Schotanus, R. Kamermans and P. Dorenbos. IEEE TNS 37 (1990) 177

[18] A.V. Gektin, N.V. Shiran, Proceedings of the International Conference on Inorganic Scintillators and Their Applications : SCINT 97 : Shanghai, P.R. China, , 1997, page 115

[19] R. T. Williams, J. Q. Grim, Qi Li, K. B. Ucer, and W. W. Moses. Phys. Status Solidi B 248 (2011) 426.

[20] S. Kubota, S. Sakuragi, S. Hashimoto and J.Ruan(gen). NIM A268 (1988) 275

[21] B. P. Aduev, E. D. Aluker, G. M. Belokurov, and V. N. Shvayko, Phys. Status Solidi B 208 (1998) 137.

[22] K. Tanimura and N. Itoh Phys. Rev. Lett. 64 (1990) 1429.

[23] M. J. Weber, Phys. Rev. B 8 (1973) 54.

[24] R. T. Williams, K. B. Ucer, J. Q. Grim, K. C. Lipke, L. M. Trefilova, W. W. Moses, IEEE TNS, 57 (2010) 1187.

[25] A. N. Belsky, P. Chevallier, P. Dhez, P. Martin, C. Pédrini, A. N. Vasil'ev, Nuclear Instruments and Methods in Physics Research A361 (1995) 384. 


\section{Figure captions}

Fig.1. Variation of FWHM of laser spot on sample surface vs relative lens position $Z$.

Fig. 2. Emission spectra of pure CsI under different excitation densities. Maximum densities in the excited domain are: $1-10^{17} \mathrm{~cm}^{-3}(\mathrm{Z}=-75 \mathrm{~mm}) ; 2-10^{18} \mathrm{~cm}^{-3}(\mathrm{Z}=-40 \mathrm{~mm}) ; 3-10^{20} \mathrm{~cm}^{-3}(\mathrm{Z}=15 \mathrm{~mm})$.

Fig.3 Left panel: Z-scan curves for FIL emission measured using laser beam of three pulse energies: 2 $\mathrm{nJ}, 5 \mathrm{~nJ}$ and $30 \mathrm{~nJ}$. Right panel: Z-scan curves for the two emission bands of CsI measured at $5 \mathrm{~nJ}$. The focal point of the lens is in the sample plane at $\mathrm{Z}=17 \mathrm{~mm}$.

Fig.4 Averaged light yield $\left\langle\eta_{\text {lum }}\right\rangle\left(n_{\text {max }}\right)$ the 2.9-eV (blue) and 4.1-eV FIL (red) bands as a function of the maximum excitation density induced by the laser. Solid lines correspond to the fitting procedure described in the text.

Fig. 5. Reconstructed dependence of luminescence LY $\eta_{\text {lum }}(n)$ of FIL (red) and $2.9 \mathrm{eV}$ (blue) bands as a function of uniform density $n$ of electronic excitations.

Fig. 6 Decay of the 2.9-eV band at $\mathrm{n}_{\max }=10^{18} \mathrm{~cm}^{-3}(1-$ green dots $)$ and $5 \times 10^{20} \mathrm{~cm}^{-3}(2$ - orange dots $)$.

Fig. 7. FIL decay curves at different excitation densities $n_{\max }: 1-10^{17} \mathrm{~cm}^{-3}, 2-10^{18} \mathrm{~cm}^{-3}, 3-10^{19} \mathrm{~cm}^{-}$ ${ }^{3}, 4-10^{20} \mathrm{~cm}^{-3}, 5$ - аппаратная функция детектора. Dots correspond to experimental data, solid lines are fits by 2 or 3 exponentials.

Fig. 8.1 and 2 - decay of $2.9 \mathrm{eV}$ band, 3 and 4 - decay of the FIL band, excited by 15-nJ laser pulses at $10^{18} \mathrm{~cm}^{-3}$ for curves 2 and 4 , at $5 \times 10^{20} \mathrm{~cm}^{-3}$ for curves 1 and 3 . For comparison, curve 1 and 2 were superimposed with curves 3 and 4, the result is the curves 1' and 2'.

Fig 9. Luminescence excitation spectra of FIL (dots 2, left axis) and $2.9 \mathrm{eV}$ band (curve 1, small right axis) in CsI in the region of $\mathrm{L}_{3}$ edge of I and excitation spectrum of corresponding X-ray fluorescence (curve 3).

Table 1 Approximation of FIL decay curves from figure 7 by 2 or 3 exponential function.

\begin{tabular}{|l|l|l|l|l|l|l|}
\hline $\begin{array}{l}\text { Density, } \\
\mathrm{cm}^{-3}\end{array}$ & $\mathrm{t} 1, \mathrm{~ns}$ & $\%$ & $\mathrm{t} 2$ & $\%$ & $\mathrm{t} 3$ & $\%$ \\
\hline $10^{17}$ & 0.36 & 88.4 & 6.0 & 11.6 & - & - \\
\hline $10^{18}$ & 0.60 & 76.6 & 6.07 & 23.4 & - & - \\
\hline $10^{19}$ & 1.08 & 49.7 & 5.33 & 42.6 & 15.1 & 7.7 \\
\hline $10^{20}$ & 0.68 & 31 & 2.87 & 27 & 11.7 & 42 \\
\hline
\end{tabular}

